\title{
Involvement of CELSR3 Hypermethylation in Primary Oral Squamous Cell Carcinoma
}

\author{
Goot Heah Khor ${ }^{1,2 *}$, Gabrielle Ruth Anisah Froemming ${ }^{3}$, Rosnah Binti Zain ${ }^{4}$, \\ Thomas Mannil Abraham ${ }^{5}$, Thong Kwai Lin ${ }^{6}$
}

\begin{abstract}
Background: Promoter hypermethylation is a frequent epigenetic mechanism for gene transcription repression in cancer and is one of the hallmarks of the disease. Cadherin EGF LAG seven-pass G-type receptor 3 (CELSR3) contributes to cell contact-mediated communication. Dysregulation of promoter methylation has been reported in various cancers. Objectives: The objectives of this study were to investigate the CELSR3 hypermethylation level in oral squamous cell carcinomas (OSCCs) using methylation-sensitive high-resolution melting analysis (MS-HRM) and to correlate CELSR3 methylation with patient demographic and clinicopathological parameters. Materials and Methods: Frozen tissue samples of healthy subjects' normal mucosa and OSCCs were examined with regard to their methylation levels of the CELSR3 gene using MS-HRM. Results: MS-HRM analysis revealed a high methylation level of CELSR3 in $86 \%$ of OSCC cases. Significant correlations were found between CELSR3 quantitative methylation levels with patient ethnicity $(P=0.005)$, age $(P=0.024)$ and pathological stages $(P=0.004)$.A moderate positive correlation between CELSR3 and patient age was also evident $(R=0.444, P=0.001)$. Conclusions: CELSR3 promoter hypermethylation may be an important mechanism involved in oral carcinogenesis. It may thus be used as a biomarker in OSCC prognostication.
\end{abstract}

Keywords: CELSR3 - hypermethylation - oral squamous cell carcinoma - biomarker

Asian Pac J Cancer Prev, 17 (1), 219-223

\section{Introduction}

Cadherin EGF LAG seven-pass G-type receptor 3 (CELSR3), belongs to a flamingo subfamily, part of the cadherin superfamily. It is a member of cell contactmediated communication, with cadherin domains acting as homophilic binding regions and EGF-like domains involved in cell adhesion and receptor-ligand interactions. Its dysregulation of DNA methylation has been reported recently in various cancer types including renal, bladder, hepatic and pancreatic carcinomas (Erka et al., 2010; Morris et al., 2010; Jeong et al., 2011; Shen et al., 2012). Together, these data suggest an important role for CELSR3 in cancerous cells that warrants further investigation. However, the specific role of methylation of CELSR3 has not been determined up to date. Further work will be required to understand its role in OSCC pathogenesis. To our knowledge, CELSR3 is a novel gene involved in epigenetic inactivation, which has not been reported elsewhere in OSCC.
Oral cancer is the sixth most common malignancy worldwide, and approximately $90 \%$ of oral cancers are predominantly OSCC (Jemal et al., 2007). Cancer progression is considered a multiple process which involves a number of aberrant genetic and epigenetic events culminating in malignant transformation. However, epigenetic aberrance plays a vital role in many types of cancer progression (Baylin et al., 2006; Mascolo et al., 2012; Tian et al., 2015). DNA methylation, the most widely studied epigenetic mechanism occurs in $\mathrm{CpG}$ islands that are commonly located in the gene promoter regions. Promoter hypermethylation is a frequent epigenetic mechanism for silencing gene transcription in cancer and is regarded as one of the hallmarks of carcinogenesis (Herman and Baylin, 2003).

Analysis of DNA methylation is a promising tool for early cancer detection, risk assessment, and response to gene therapy (Einav Nili et al., 2008). Methylation analysis supported the proposal that gene transcription silencing by CELSR3 hypermethylation in various types

${ }^{I}$ Centre of Preclinical Science Studies, Faculty of Dentistry, Universiti Teknologi MARA, Sungai Buluh Campus, ${ }^{2}$ Health and Wellbeing Community of Research Group, Universiti Teknologi MARA, Shah Alam, ${ }^{3}$ Institute of Pathology, Laboratory and Forensic Medicine, Faculty of Medicine, Universiti Teknologi MARA, Sungai Buluh Campus, ${ }^{4}$ Oral Cancer Research and Coordinating Centre, Faculty of Dentistry, University of Malaya, Kuala Lumpur, ${ }^{5}$ Tengku Ampuan Rahimah Hospital, Department of Oral and Maxillofacial Surgery, Ministry of Health Malaysia, Klang, ${ }^{6}$ Institute of Biological Science, Faculty of Science, University of Malaya, Kuala Lumpur, Malaysia.*For correspondence: gootheah@salam.uitm.edu.my 
of cancers, which could serve as a potential biomarker for cancer detection. Thus, quantitative high-resolution melting analysis (HRM) analysis was applied for quantifying promoter methylation of CELSR3 gene in OSCC patients for the study (Amornpisutt et al., 2012; Wojdacz et al., 2008). HRM analysis is an emerging and advanced quantitative technique for detection of nucleic acid sequence variation in cell mutations and single nucleotide phenomenon as well as DNA methylation studies (Reed et al., 2007). Its principle is based on bisulfite-treated DNA templates with different contents of methyl cytosine can be distinguished differences in melting temperatures. It provides a sensitive detection of methylation levels in a labor- and cost-efficient manner, since it does not require expensive probes and reference gene assays for normalization (Wittwer 2009). In addition, status of imprinted loci as well as identification of heterogeneous methylation can be investigated. With HRM, all CpGs regions within the amplicon are analyzed and distinguished by the melting curve pattern. Recent combination of new technology with DNA intercalating dyes such as EvanGreen that can be used at saturating concentrations allow the discrimination of sequence changes in PCR amplicons without manual handling separately for PCR products (Wojdacz et al., 2008).

In this study, CELSR3 gene was screened and selected as a signature candidate in promoter hypermethylation based on our previous study (Khor et al., 2013; Khor et al., 2014). This hypermethylated gene was shown to be associated with disease progression. The relationship between this signature gene and OSCC patients' demographic and clinicopathological parameters were further investigated by statistical analysis.

\section{Materials and Methods}

\section{Samples}

Human genomic DNA of snapped frozen tissues from years between 2007-2010 of 4 normal tissue surrounding impacted tooth of healthy individuals and 46 OSCC were obtained in the present study. All of these selected patients were diagnosed as primary OSCC by the oral pathogist, and their consents were obtained prior to the study. They were evaluated and enrolled in appropriate protocols in the joint network of the Malaysian Oral Cancer Database and Tissue Bank System (MOCDTBS) coordinated by Oral Cancer Research Coordinating Center (OCRCC) after obtaining subject consent following appropriate ethical committee approval from Medical Ethics Commitee of the Faculty of Dentistry, Universiry of Malaya (Ethic reference no: DF OP1101/0049(L)).

\section{DNA extraction}

About 500 ng of extracted-DNA of snaped frozen tissues was obtained from OCRCC. The protocol is as described: Briefly, a total of $0.1 \mathrm{mg}$ snapped frozen specimen was sectioned using Leica CM 1850 Cryostat (Leica, Deerfield, IL) at temperature of $20^{\circ} \mathrm{C}$. The representative section was sectioned for Haematoxylin and Eosin evaluations, to ensure a minimum of $70 \%$ of tumour cells by the oral pathologist. The selected specimens were then proceeded for DNA extraction using QIAamp DNA Blood Mini Kit (QIAGEN GmbH, Hilden, Germany) followed the manufacturer's procedures. Concentration and purity of DNA samples were determined with a Nanodrop 1000 spectrophotometer. (NanoDrop Technologies, Wilmington, DE, USA). DNA quality was evaluated-based on OD260/280 and the integrity was also checked by $1 \%$ agarose gel electrophoresis analysis. The genomic DNA were frozen and stored at $-20^{\circ} \mathrm{C}$.

\section{Bisulfite-converted DNA}

The protocol was processed according the manufacturer's description (EpiTect, QIAGEN GmbH, Hilden, Germany). Briefly, $1 \mathrm{ng}-2 \mu \mathrm{g}$ of genomic DNA is incubated with a sodium bisulfite buffer in a thermocycler with alternating cycles of $95^{\circ} \mathrm{C}$ and $60^{\circ} \mathrm{C}$, through steps of denaturation, sulphonation and deamination of cytosine to uracil. Afterwards, the DNA is bound again to a silica membrane and then washed and incubated with a desulphonation buffer and eluted with elution buffer. The bisulfite-converted DNA products were quantified using a Nanodrop 1000 spectrophotometer.

\section{Design of HRM primers}

Several factors were taken into considerations during the primer design for this study to avoid PCR bias (Wojdacz et al., 2009). Briefly, primers were designed with the following conditions: i) primers must contain at least one $\mathrm{CpG}$ dinucleotides as near as possible to the 5' end of forward primer, and ii) at the 3' end of each primer must contain one or more natural thymidine nucleotides (T) originating from a non- $\mathrm{CpG}$ cytosine. Inclusion of a CpG dinucleotide in the 5' end of the primer permits experimental manipulation of the PCR bias towards the methylated DNA. The criterion of natural thymidine inclusion is to amplify only bisulfite-modified template DNA.

In this study, CELSR3 primer was designed in the promoter region using Methyl Primer Express Software v1.0 (Applied Biosystems Inc. Foster City, CA, USA). The primers for unmethylated and methylated gene promoter regions are: Forward primer: 5' GTTAGGTTCGTGTTGTTGGAT 3'. Reverse primer: 5' CTTTACCATCGACAACCTCA 3'. The melting temperature was $52^{\circ} \mathrm{C}$, amplicon length $135 \mathrm{bp}$ and locus in genome browser UCSC: chr3:48698335-48701667.

\section{High resolution melting (HRM) analysis}

HRM analysis was performed on the thermal cycler (CFX 96, Real-Time System C1000 Thermal Cycler, BioRad, Hercules, USA). PCR protocol was run according to manufacturer's protocol (Bio-Rad, Hercules, USA). Briefly, PCR was performed in a $10 \mu 1$ volume of Precision Melt Supermix, $200 \mathrm{nM}$ of each primer, and $10 \mathrm{ng}$ bisulfite-treated DNA templates. Each reaction was run with triplicate. The PCR and HRM conditions are as follows: initial DNA denaturation at $95^{\circ} \mathrm{C}$ for 2 min, followed by 45 cycles of denaturation step at $95^{\circ} \mathrm{C}$ for $10 \mathrm{sec}$, annealing and extension steps at $52^{\circ} \mathrm{C}$ for 30 sec and $72^{\circ} \mathrm{C}$ for $30 \mathrm{sec}$, followed by one HRM cycle of heteroduplex formation step at $95^{\circ} \mathrm{C}$ for $30 \mathrm{sec}, 60^{\circ} \mathrm{C}$ for 
$1 \mathrm{~min}$ and high resolution melting step at $65^{\circ} \mathrm{C}$ to $95^{\circ} \mathrm{C}$ in $0.2^{\circ} \mathrm{C}$ increments for $10 \mathrm{sec}$. HRM standard melting curve that derived from preparation of dilution of fully methylated DNA into unmethylaed DNA by ratios of methylation DNA: $0 \%, 25 \%, 50 \%, 75 \%$ and $100 \%$, was included in each run.

The normalized and difference graphs were used to analyze the data. The normalized graph was generated by the monitoring of dissociation of the fluorescent dye from double-stranded DNA as the temperature increased (Wojdacz et al., 2008). The EvanGreen dye used in this study can only fluoresce when it is intercalated into double-strand DNA. The normalized graph shows the degree of reduction in fluorescence over a temperature range (typically $65^{\circ} \mathrm{C}$ to $95^{\circ} \mathrm{C}$ in $0.2^{\circ} \mathrm{C}$ increments). All samples including the normal control were plotted according to their melting profiles. In the difference graph, the melting profiles of each sample were compared to methylation control.

\section{Statistical analysis}

HRM data were analyzed using Precision Melt Analysis Software (Bio-Rad Hercules, USA), output of the plots showed as normalized melting curves and difference plots. Categorical variables were performed using Chi Square test or Fisher's exact test and independent samples T test using SPSS for Windows software (version 17.0; SPSS Inc., Chicago, IL, USA). Bivariate correlate test to determine whether there is a significant correlation between patients' demographic and clinicopathological parameters with CELSR3 quantitative methylation levels. The level of significance was taken to be $\mathrm{p}$ value $<0.05$.

\section{Results}

Profiling of methylation levels of CELSR3 in normal tissues and tumour samples

The consistency of CELSR3 methylation was observed in the normalized and different melting profiles that derived from samples with different ratios of methylated and unmethylated templates. The normalized melting profiles of the PCR product amplified from the same mix of methylated and unmethylated template were consistent between replicates and between different runs samples. The consistency of HRM profiles allows the estimation of the methylation content of unknown samples on the basis of similarities of normalized HRM profiles.

\section{Methylation-specific high resolution melting analysis for percentage of CELSR3 methylation level}

In HRM analysis, methylation percentage of samples was obtained by comparing with the HRM standard melting curve (Figure 1). In the study, range of $0-25 \%$ of CELSR methylation levels was observed in 4 normal mucosal tissues. Overall, three (6\%) of cases showed range of $0-25 \%$, five $(10 \%)$ cases with range of $25-50 \%$, four (8\%) cases with range of 50-75\% and thirty-four (68\%) cases with range of 75-100\% methylation levels in tumour tissues (Figure 2). The level of methylation was considered high if the methylation percentage is more than $50 \%$. Thus, eight $(16 \%)$ cases were reported as low and thirty-eight
$(76 \%)$ cases were considered as high methylation levels, respectively in the study.

Demographic and clinicopathological parameters of OSCC and CELSR3 hypermethylation

Table 1 demonstrated the demographic and clinicopathologic parameters of the OSCC patients. Characteristics of age, gender, ethnic group, habits of alcohol drinking, tobacco smoking and betel-quid chewing, tumour sites, pathological stages and lymph node metastasis (LNM) status were included in the study. The ethnic of studied groups consisting of 25 men and 25 women, with a median age of 56.9 years (age range of 20-87 years) were Malays $(n=12)$, Chinese $(n=15)$ and Indians $(n=23)$. Most of the patients $(72 \%)$ have habits of alcohol drinking, tobacco smoking and/or betel-quid chewing while only $28 \%$ of the patients have none of the aforementioned habits. For tumour site, $19(38 \%)$ were from buccal mucosa, $16(32 \%)$ gum and $15(30 \%)$ tongue. Four patients $(8 \%)$ demonstrated no pathological TNM stage, $7(14 \%)$ had stage I, $6(12 \%)$ stage II, $13(26 \%)$ stage III and $20(40 \%)$ stage IV of pathological TNM stages. LNM data revealed $28(56 \%)$ of no metastases and $22(44 \%)$ of metastases cases.

A statistically significant association was found between CELSR3 hypermethylation with patients' ethnicity $(\mathrm{P}=0.005)$, age $(\mathrm{P}=0.024)$ and pathological stages $(\mathrm{P}=0.004)$ using Chi Square or Fisher Exact tests of SPSS version 17.0. A moderate strength of positive correlation between CELSR3 and patients' age was associated in the study $(\mathrm{R}=0.444, \mathrm{P}=0.001)$. There was

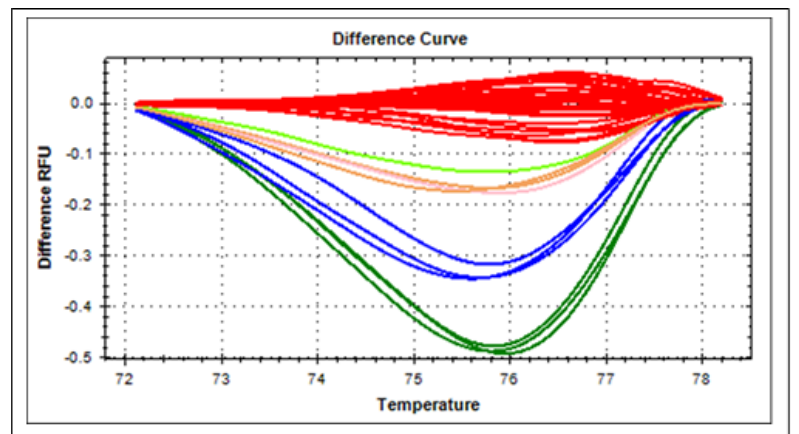

Figure 1. Representative Difference Plot of Standard and Samples of CELSR3. Most of the representative samples show $100 \%$ methylation level. (Indicator: Red coloured line represented $100 \%$ methylation, light green coloured line is $75 \%$, brown coloured line is $50 \%$, blue coloured line is $25 \%$ and dark green coloured line is $0 \%$ )

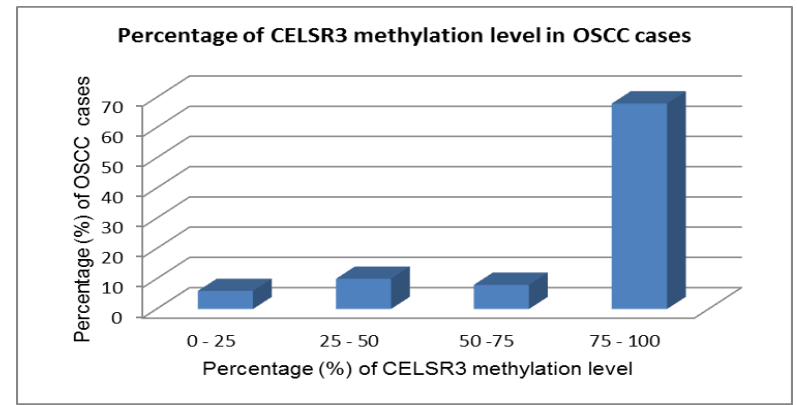

Figure 2. Percentage of CELSR3 Methylation Level in OSCC Cases 
Table 1. Relationships between Demographic and Clinicopathological Parameters with CELSR3 Methylation Level

\begin{tabular}{|c|c|c|c|c|c|c|c|}
\hline \multirow[t]{2}{*}{ Characteristics } & & \multicolumn{2}{|c|}{ Frequency $(\%)$} & \multicolumn{3}{|c|}{ CELSR3 (\% of methylation) } & \multirow{2}{*}{$\begin{array}{c}\text { Chi Square and Fisher's } \\
\text { Exact test (P value) }\end{array}$} \\
\hline & & & $0-25$ & $25-50$ & $50-75$ & $75-100$ & \\
\hline \multirow[t]{2}{*}{ Gender } & Male & $25(50 \%)$ & 3 & 2 & 2 & 18 & \\
\hline & Female & $25(50 \%)$ & 3 & 4 & 2 & 16 & 0.893 \\
\hline \multirow[t]{3}{*}{ Ethnic } & Malay & $12(24 \%)$ & 1 & 1 & 1 & 9 & \\
\hline & Chinese & $15(30 \%)$ & 3 & 2 & 1 & 9 & \\
\hline & Indian & $23(46 \%)$ & 2 & 3 & 2 & 16 & $0.005^{*}$ \\
\hline \multirow[t]{2}{*}{ Age (Years) } & $<50$ & $14(28 \%)$ & 5 & 1 & 1 & 7 & \\
\hline & $>50$ & $36(72 \%)$ & 1 & 5 & 3 & 27 & $0.024 *$ \\
\hline \multirow[t]{5}{*}{ Habits } & No habit & $14(28 \%)$ & 4 & 1 & 1 & 8 & \\
\hline & Betell quid chewing & $16(32 \%)$ & 1 & 2 & 1 & 12 & \\
\hline & Tobacco \& smoking & $6(12 \%)$ & 0 & 1 & 0 & 5 & \\
\hline & Alcohol drinking & $2(4 \%)$ & 0 & 1 & 0 & 1 & \\
\hline & More than 2 habits & $12(24 \%)$ & 1 & 1 & 2 & 8 & 0.753 \\
\hline \multirow[t]{3}{*}{ Tumors Site } & Buccal mucosa & $19(38 \%)$ & 1 & 2 & 1 & 15 & \\
\hline & Gum & $16(32 \%)$ & 4 & 1 & 1 & 10 & \\
\hline & Tongue & $15(30 \%)$ & 1 & 3 & 2 & 9 & 0.465 \\
\hline \multirow[t]{5}{*}{ Pathological TNM stages } & 0 & $4(8 \%)$ & 4 & 0 & 0 & 0 & \\
\hline & I & $7(14 \%)$ & 0 & 1 & 1 & 5 & \\
\hline & II & $6(12 \%)$ & 0 & 0 & 1 & 5 & \\
\hline & III & $13(26 \%)$ & 1 & 1 & 2 & 9 & \\
\hline & IV & $20(40 \%)$ & 1 & 4 & 0 & 15 & $0.004 *$ \\
\hline \multirow[t]{2}{*}{ LNM } & No & $28(56 \%)$ & 4 & 3 & 3 & 18 & \\
\hline & Yes & $22(44 \%)$ & 2 & 3 & 1 & 16 & 0.537 \\
\hline
\end{tabular}

*Significant correlation between CELSR3 methylation levels with OSCC patients' ethnicity ( $\mathrm{P}=0.005)$, age ( $\mathrm{P}=0.024)$ and pathological TNM stages $(\mathrm{P}=0.004)$

no statistical significance of association between the CELSR3 methylation with patients' gender, habits, LNM and tumour sites.

\section{Discussion}

DNA methylation plays an essential role in maintaining cellular function, and methylation aberrations may lead to carcinogenesis. Thus, several methods have been developed recently to analyse genes' methylation status in human cells, depending on their detection strengths and weaknesses. One of these methods is the methylationspecific PCR (MS-PCR which applies specific designed methylated and unmethylated primers for qualifying the methylation level. It is a sensitive but not quantitative technique for the methylation level (Wojdacz and Dobrovic, 2007; Montgomery et al., 2010). On the other hand, the bisulfite sequencing is a gold standard technique for methylation level quantitation, but it is relatively insensitive and not cost effective if it is applied as a screening test (Wojdacz et al., 2010; Khor et al., 2013). Advances in the DNA melting technologies combined with PCR-based methylation detection application, led to the development of a novel technique, MS-HRM (Wojdacz et al., 2010). The MS-HRM provides some advantages that overcome the weaknesses of previous methods and it provides high throughput and is very cost effective in quantifying single site gene-specific DNA methylation (Wojdacz et al., 2010). The estimation of unknown methylation level can be done by comparing a series of dilution of standard melting curve, which derived from varying mixtures of methylated and unmethylated DNA standards (Dobrowolsk and Wittwer 2011; Wojdacz et al.,
2010). In this study, we found that MS-HRM can be used as a sensitive technique in quantifying the methylation level for OSCC samples.

CELSR3 is a member of the flamingo protein subfamily which is part of the cadherin superfamily. The flamingo cadherins have nine cadherin domains, seven epidermal growth factor-like repeats and two laminin A G-type repeats. It is postulated that these proteins are receptors involved in contact-mediated communication, with cadherin domains acting as homophilic binding regions and the EGF-like domains involved in cell adhesion and receptor-ligand interactions. CELSR3 is crucial in controlling neural development which develop and maintain individual plastic changes throughout life by regulating planar cell polarity (PCP) through interactions with PCP proteins in WNT/PCP signaling pathway (Tissir et al., 2005; Feng et al., 2012). WNT/ PCP signaling pathway is implicated in embryogenesis and carcinogenesis. Aberrant activation of the WNT/PCP signaling pathway leads to malignancy through abnormal tissue polarity, invasion, and metastasis (Katoh, 2005). Its' up-regulation in pancreatic cancer could provide as a potential druggable target since its protein is located at the plasma membrane and has intriguing capabilities in WNT/PCP signaling pathway (Katoh, 2005). In addition, Erkan et al (2010) found that in pancreatic and hepatic carcinoma, CELSR3 was identified as a hypermethylated marker as reported in our OSCC cases (Khor et al., 2013). Moreover, Katoh and Katoh (2007) demonstrated CELSR3 expression is reduced in colorectal cancer which showed gene expression is undergo inactivation effect by hypermethylation. Thus, we predict that hypermethylation mechanism has reduced the gene expression in OSCC 
cases. CELSR3 hypermethylation status in our study suggested that it may involve in oral carcinogenesis. To the best of our knowledge, this is the first time CELSR3 is being investigated in our OSCC cases.

The statistical significance was found in relation of CELSR3 with patients' ethnicity, age and pathological stages in the study. Similar findings also reported by Cianfrocca et al (2004), where patients' ethnicity, age and pathological stage for prognostication may be used to identify a group of early-stage breast cancer patients who have a higher risk recurrence. In addition, Pimenta Amaral et al (2004) group found that tumour from floor of the mouth with the histological grade II and III has a higher risk of having occult metastasis than grade I. In the study, CELSR3 hypermethylation may associate with age-related epigenetic mechanism in OSCC cases. This implies that CELSR3 hypermethylation can be used as a prognostic tool in OSCC patient management in term of patients' ethnicity, age and pathological stage.

In conclusion, CELSR3 hypermethylation was detected quantitatively by MS-HRM in this study. Overall our results suggest that CELSR3 promoter hypermethylation is an important mechanism involved in oral carcinogenesis and can be used as prognostic tool for OSCC patients. However, a larger OSCC cohort is needed to validate results and subsequent biological analysis of the proteins encoded by this gene may lead to a better understanding of oral carcinogenesis.

\section{Acknowledgements}

This work were financed by the grant of $600-\mathrm{RMI} /$ DANA/5/3/RIF (633/2012) of Universiti Teknologi MARA, Malaysia and 600/RMI/FRGS 5/3 (25/2015) of Fundamental Research Grant Scheme of the Ministry of Higher Education, Malaysia. We appreciated the Oral Cancer Research and Coordinating Centre (OCRCC), University of Malaya to provide the oral cancer tissues and data from the Malaysia Oral Cancer Database and Tissue Bank System (MOCDTBS).

\section{References}

Amornpisutt R, Sriraksa R, Limpaiboon T (2012). Validation of methylation-sensitive high resolution melting for the detection of DNA methylation in cholangiocarcinoma. Clin Biochem, 45, 1092-4.

Baylin SB, Ohm JE (2006). Epigenetic gene silencing in cancera mechanism for early oncogenic pathway addiction? Nature Rev Cancer, 6, 107-16.

Cianfrocca M, Goldstein LJ (2004). Prognostic and predictive factors in early-stage breast cancer. The Oncologist, 9 , 606-16.

Dobrowolski SF, Wittwer CT (2011). High-resolution melt profiling, in molecular analysis and genome discovery. John Wiley \& Sons, Ltd, p. 81-113.

Einav Nili GY, Saito Y, Egger G, et al (2008). Cancer epigenetics, modifications, screening, and therapy. Аnnu Rev Med, 59, 267-80.

Erkan M, Weis N, Pan Z, et al (2010). Organ-, inflammation-and cancer specific transcriptional fingerprints of pancreatic and hepatic stellate cells. Mol Cancer, 9, 88-103.

Feng, J, Han Q, Zhou L (2012). Planar cell polarity genes,
Celsr1-3 in neural development. Neurosci Bulletin, 28, 39-15.

Herman JG, Baylin SB (2003). Gene silencing in cancer in association with promoter hypermethylation. New Engl J Med, 349, 2042-54.

Jemal A, Siegel R, Ward E (2007). Ca_statistica. CA Cancer J Clin, 57, 43-66.

Jeong P, Ha YS, Cho IC, et al (2011). Three-gene signature predicts disease progression of non-muscle invasive bladder cancer. Oncol Lett, 2, 679-84.

Katoh M (2005). WNT/PCP signaling pathway and human cancer (Review). Oncol Reports, 14, 1583-8.

Katoh M, Katoh M (2007). Comparative integromics on noncanonical WNT or planar cell polarity signaling molecules, Transcriptional mechanism of PTK7 in colorectal cancer and that of SEMA6A in undifferentiated ES cells. Inter $J$ Mol Med, 20, 405-9.

Khor GH, Froemming GRA, Zain RB, et al (2013). DNA methylation profiling revealed promoter hypermethylationinduced silencing of p16, DDAH2 and DUSP1 in primary oral squamous cell carcinoma. Inter J Med Sci, 10, 1727-39.

Khor GH, Froemming GRA, Zain RB, et al (2014). Screening of differential promoter hypermethylated genes in primary oral squamous cell carcinoma. Asian Pacific J Cancer Prev, 15, 8957-61.

Mascolo M, Siano M, Ilardi G, et al (2012). Epigenetic disregulation in oral cancer. Int J Mol Sc, 13, 2331-53.

Montgomery JL, Sanford LN, Wittwer CT (2010). Highresolution DNA melting analysis in clinical research and diagnostics. Expert Rev Mol Diagn, 10, 219-40.

Morris MR, Ricketts CJ, Gentle D, et a (2010). Genome-wide methylation analysis identifies epigenetically inactivated candidate tumour suppressor genes in renal cell carcinoma. Oncogene, 30, 1390-401.

Pimenta Amaral TM, da Silva Freire AR, Carvalho AL, et al (2004). Predictive factors of occult metastasis and prognosis of clinical stages I and II squamous cell carcinoma of the tongue and floor of the mouth. Oral Oncol, 40, 780-6.

Reed GH, Kent JO, Wittwer CT (2007). High-resolution DNA melting analysis for simple and efficient molecular diagnostics. Pharmacogenomics, 8, 597-608.

Shen J, Wang S, Zhang YJ, et al (2012). Genome wide DNA methylation profiles in hepatocellular carcinoma. Hepatology, 55, 1799-808.

Tian XY, Zhang L, Sun LG, et al (2015). Epigenetic Regulation of miR-129-2 Leads to Overexpression of PDGFRa and FoxP1 in Glioma Cells. Asian Pac J Cancer Prev, 16, 6129-33.

Tissir F, Bar I, Jossin Y, et al (2005). Protocadherin Celsr3 is crucial in axonal tract development. Nature Neurosci, 8, 451-7.

Wittwer CT (2009). High-resolution DNA melting analysis. advancements and limitations. Hum Mutat, 30, 857-9.

Wojdacz TK, Dobrovic A (2007). Methylation-sensitive high resolution melting (MS-HRM), a new approach for sensitive and high-throughput assessment of methylation. Nucleic Acids Res, 35, 41.

Wojdacz TK, Dobrovic A, Hansen LL (2008). Methylationsensitive high-resolution melting. Nature Protoc, 3, 1903-8.

Wojdacz TK, Borgbo T, Hansen LL (2009). Primer design versus PCR bias in methylation independent PCR amplifications. Epigenetics, 4, 231-4.

Wojdacz TK, Møller TH, Thestrup BB, et al (2010). Limitations and advantages of MS-HRM and bisulfite sequencing for single locus methylation studies. Expert Rev Mol Diagn, 10, 575-80. 